\title{
Twenty-one-cm Studies of Some Interstellar Clouds
}

\author{
R. D. Davies \\ Jodrell Bank Experimental Station, Manchester University, Manchester, England
}

\section{A. RELATION BETWEEN HI AND HII IN THE CYGNUS-X RADIO COMPLEX}

\section{(1) Observations}

C YGNUS-X is an extended radio source centered at $\delta=40^{\circ}$ and R.A. $=20^{h} 25^{m}\left(l=48^{\circ}, b=0^{\circ}\right)$. Its emission in the continuous spectrum has been studied by Davies ${ }^{1}$ using high resolution surveys at the following frequencies: 92,158 , and $1425 \mathrm{Mcps}^{2}{ }^{2} 242,{ }^{3} 400,{ }^{4}$ $600,{ }^{5}$ and $900^{6}$ Mcps. The background emission at the position of the source was removed by a smooth interpolation in galactic longitude between the two sides of the source. The resultant flux density did not change significantly with frequency and had the value $4.5 \times 10^{-23} \mathrm{wm}^{2}(\mathrm{cps})^{-1}$. Moreover, the brightness temperature $T_{S}$ of the center of the source varied inversely as the square of the frequency $\nu ; T_{S} \cdot \nu^{2}$ had the value $3.5 \times 10^{19}{ }^{\circ} \mathrm{K} \mathrm{sec}-2$.

The width of the source between half-intensity points is $2.5^{\circ}$ in declination and $5.5^{\circ}$ in R.A. This may be compared with the R.A. width of $10^{\circ}$ for the neutral hydrogen in the spiral arms in this direction. The source is thus located entirely within the confines of the spiral arms; it is irregular in shape, exhibiting at least three maxima.

When 21-cm hydrogen line spectra were taken at the position of Cygnus- $\mathrm{X}$ they showed a marked fall in intensity compared with those taken nearby. The difference spectrum, $T_{D}(\nu)$, at the position of the source was derived from the observed spectrum $T_{S}(\nu)$ and the expected spectrum $T_{B}(\nu) ; T_{D}(\nu)=T_{B}(\nu)-T_{S}(\nu) . T_{B}(\nu)$ was obtained by a smooth extrapolation in galactic longitude between spectra observed on either side of the source. In general, the value of $T_{D}(\nu)$ obtained when radiation from a radio source of brightness temperature $T_{S}$, passes through hydrogen of optical depth $\tau(\nu)$ is

$$
T_{D}(\nu)=T_{S}\left(1-e^{-\tau(\nu)}\right) .
$$

[For a derivation see Eq. (3) of Hagen et al. ${ }^{7}$ The

\footnotetext{
${ }^{1}$ R. D. Davies, Monthly Notices Roy. Astron. Soc. (to be published).

${ }_{2}$ Observations taken by the author at Jodrell Bank Experimental Station.

3 J. D. Kraus and H. C. Ko, Sky and Telescope 14, 22 (1954). ${ }_{4}^{4}$ Seeger, Westerhout, and van de Hulst, Bull. Astron. Soc. Neth 13,89 (1956)

5 J. H. Piddington and G. H. Trent, Australian J. Phys. 9, 481 (1956).

${ }^{6}$ Denisse, Leroux, and Steinberg, Compt. rend. 240, 278 (1955)

${ }^{7}$ Hagen, Lilley, and McClain, Astrophys. J. 122, 361 (1955) International Astronomical Union Symposium No. 4, "Radio
}

second term is negligible since the continuous opacity of the source is much less than unity.] Its maximum value is $T_{S}\left(=16^{\circ} \mathrm{K}\right.$ for Cygnus- $\mathrm{X}$ from the results given in the first paragraph). The resultant plots of $T_{D}(\nu)$ and $T_{B}(\nu)$ for the position $\delta=40.5^{\circ}$ and R.A. $=20^{h} 28^{m}$ are given in Fig. 1. The value of $T_{D}(\nu)$ for the first spiral arm is that expected when radiation (from Cygnus-X) traverses this arm, $\sim 16^{\circ}$. However, for the second arm $T_{D}(\nu)$ exceeds $T_{S}$ by a factor of two. This result cannot then be an absorption effect alone. There is no absorption in the outer arm at 10 $\mathrm{kpc}$, so the source lies nearer the sun than this distance.

This anomalous value of $T_{D}(\nu)$ in the second spiral arm was checked by taking drift curves across Cygnus- $X$ at different hydrogen-line frequencies. A difference spectrum for any part of Cygnus-X could then be synthesized. Twenty values for $T_{D}(\nu)$ and $T_{S}(\nu)$ derived in this way for $\delta=40.5^{\circ}$ and R.A. $=20^{h} 28^{m}$ are plotted as crosses in Fig. 1. The original result is confirmed.

\section{(2) Interpretation of Results}

The foregoing radio results can be explained in terms of the removal (viz., ionization) of neutral hydrogen from the position of Cygnus- $\mathrm{X}$ to form an HII complex. In this case $N_{\mathrm{H}}$, the density of neutral hydrogen removed, may be put numerically equal to $N_{e}$, the number of free electrons created. This suggestion is substantiated by the following arguments.

(a) The continuum radiation is that expected from an HII region. Its flux density spectrum does not vary

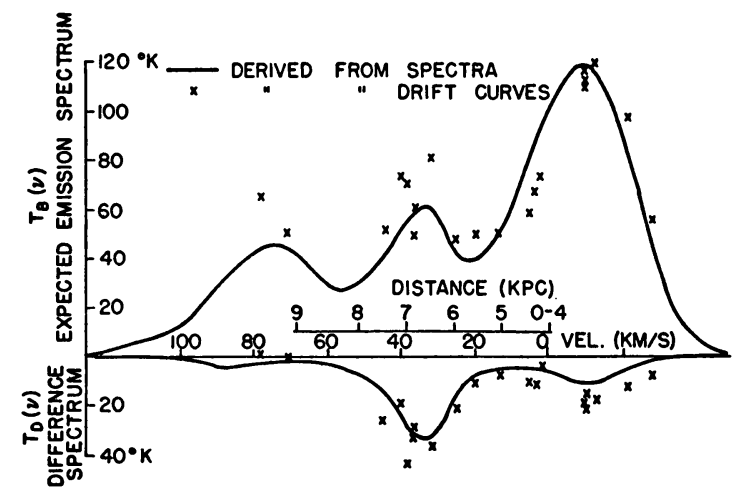

FIG. 1. Plots of $T_{D}(\nu), T_{B}(\nu)$ at $\delta=40.5^{\circ}$ R. A. $=20^{h} 28^{m}$. Full lineobtained from spectra. Crosses - obtained from drift curves.

Astronomy," (Cambridge University Press, New York, 1957), p. 81. 
with frequency. The brightness temperature of any part of the source varies inversely as the square of the frequency. The irregular shape of the source is again that expected in a large HII complex with several centers of ionization.

(b) The contours of the neutral hydrogen deficiency in the second spiral arm, $\int N_{\mathbf{H}} \cdot d l$, follow the contours of the emission from the ionized hydrogen, which is proportional to $\int N_{e}{ }^{2} \cdot d l$. This is expected on the above explanation if $N_{\mathrm{H}}$ (and thus $N_{e}$ ) do not vary markedly along the line of sight. The source thus lies at a distance of $6 \mathrm{kpc}$ if we accept the Oort rotation model.

(c) The values of $N_{e},\left(=N_{\mathrm{H}}\right)$ and $l$, the depth of the region, derived from the radio results are acceptable. Using the simplifying assumption that $N_{e}=N_{\mathrm{H}}=$ constant over a depth $l$, we can write ${ }^{8}$

$$
N_{\mathrm{H}} \cdot l=1.54 \times 10^{-5} T_{K} \cdot \eta \Delta \tau_{0}(\nu),
$$

where $l$ is measured in parsecs, $\Delta \tau_{0}(\nu)$ represents the maximum optical depth of the neutral hydrogen removed, and $\eta$ is the half-width of $T_{D}(\nu)$ in $\mathrm{cm} \mathrm{sec}^{-1}$. From the theory of free-free emission in an optically thin region, ${ }^{9}$ we have

$$
N_{e}^{2} \cdot l=1.89 \times 10^{-6} T_{e}^{\frac{1}{2}}\left(T_{S} \cdot \nu^{2}\right),
$$

where $T_{e}$ is the electron temperature. $T_{S}$ is the brightness temperature of the continuum emission from Cygnus-X at a frequency $\nu . N_{e}^{2} \cdot l$ is found from the continuum observations to be 6600 . The solution of (2) and (3) for various values of $T_{K}$ are given in Table I. These values of $l$ are directly comparable with the linear dimensions of the source $(270 \times 600 \mathrm{pc}) . N_{\mathrm{H}}$ is a factor two higher than found within other spiral arms.

(d) 22 Mcps continuum observations of this region by Burke ${ }^{10}$ show a fall in temperature at the position of Cygnus- $\mathrm{X}$. This is expected since the brightness temperature of the HII region $\left(\simeq 10^{4}{ }^{\circ} \mathrm{K}\right)$ is less than that of the background emission $\left(\simeq 25000^{\circ} \mathrm{K}\right)$.

\section{(3) Comparison with Optical Studies}

This region is the richest in the sky for $\mathrm{O}$ and $\mathrm{B}$ stars; they lie beyond the Cygnus rift which is at 700 parsecs. Although the region is heavily obscured by dust clouds the visible stars cataloged by Hiltner and

TABLE I.

\begin{tabular}{rcc}
\hline $\begin{array}{c}T_{K} \\
\left({ }^{\circ} \mathrm{K}\right)\end{array}$ & $\begin{array}{c}N_{e}=N_{\mathrm{H}} \\
\left(\mathrm{cm}^{-8}\right)\end{array}$ & $\begin{array}{c}l \\
\text { (parsecs) }\end{array}$ \\
\hline 80 & 4.6 & 310 \\
100 & 6.5 & 160 \\
125 & 7.8 & 110 \\
\hline
\end{tabular}

8 D. S. Heeschen, Astrophys. J. 121, 569 (1955).

${ }^{9}$ S. F. Smerd and K. C. Westfold, Phil. Mag. 40, 831 (1949).

${ }^{10}$ B. F. Burke, Astron. J. 61, 167 (1956).

\begin{tabular}{|c|c|c|c|c|c|}
\hline HII region & $\begin{array}{c}T_{s} \\
\left({ }^{\circ} \mathrm{K} \text { at }\right. \\
1400 \\
\text { Mcps })\end{array}$ & Reference & $\begin{array}{c}\text { Half- } \\
\text { power } \\
\text { diameter } \\
\text { (parsecs) }\end{array}$ & $\begin{array}{c}\text { Emis- } \\
\text { sion } \\
\text { measure } \\
(\text { radio) } \\
\times 10^{-3}\end{array}$ & $\underset{\left(\mathrm{cm}^{-2}\right.}{N_{\mathrm{e}}}$ \\
\hline Cygnus- $X$ & 16 & Present paper & 200 & 7 & 5 \\
\hline $\begin{array}{l}\text { Orion Nebula } \\
\text { N.G.C. } 6618\end{array}$ & 400 & $(12,13,14)$ & 1.8 & 140 & 260 \\
\hline $\begin{array}{l}\text { Omega Nebula } \\
\text { N.G.C. } 6357 \\
\text { Orion (Great arc }\end{array}$ & $\begin{array}{r}2000 \\
350\end{array}$ & $\begin{array}{l}(12,13,14) \\
(13,14)\end{array}$ & $\begin{array}{l}2.6 \\
1\end{array}$ & $\begin{array}{l}700 \\
110\end{array}$ & $\begin{array}{l}520 \\
300\end{array}$ \\
\hline $\begin{array}{l}\text { Orion (Great arc } \\
\text { of Barnard) }\end{array}$ & $\cdots$ & $\begin{array}{l}\text { (a rough estimate from } \\
\text { optical brightness) }\end{array}$ & (100) & (2) & (5) \\
\hline
\end{tabular}

TABLE II.

Johnson ${ }^{11}$ which lie within the envelope of Cygnus-X can produce an appreciable amount of the ionization required. The total ionizing capacity of these stars, $\int N_{e}{ }^{2} d(\mathrm{vol})$, was estimated from Strömgren's ${ }^{12}$ theory and comes to $3.7 \times 10^{63} \mathrm{~cm}^{-3}$. An estimate of $\int N_{e}^{2} d(\mathrm{vol})$ can also be made from the flux density of the source and it may be shown that

$$
\begin{aligned}
\int N_{e}^{2} d(\mathrm{vol}) & =\frac{c^{2} T_{e}^{\frac{1}{2}}}{0.354 k} D^{2} \cdot S \\
& =7.1 \times 10^{63} \text { if at } 3 \mathrm{kpc}, \\
& =2.8 \times 10^{64} \text { if at } 6 \mathrm{kpc},
\end{aligned}
$$

where $D$ is the distance of source and $S$ is its flux density.

The remaining ionization would need to be accounted for by obscured stars (principally in the following half of the source). An early type O-star cluster called VI Cygni lies at $\delta=41^{\circ}$ and R.A. $=20^{h} 29^{m}$. It is provisionally placed at $2 \mathrm{kpc}$ and shows heavy obscuration (up to 7 magnitudes). The 20 members so far classified provide $70 \%$ of the ionization produced at this point of Cygnus-X (which shows a secondary maximum).

\section{Conclusion}

The radio evidence is shown to confirm the suggestion that Cygnus-X is a vast HII region. Hydrogen does not appear to exist in the neutral state within the region, and it is conspicuous as a fall in the $21-\mathrm{cm}$ emission at the position of the second spiral arm. The optical and radio distances do not agree closely; however, neither value is particularly accurate for this section of the sky. The results indicate that the hydrogen density throughout this HII region is a factor of at least two higher than that within a typical spiral arm. High densities $\left(N_{e} \gg 1\right)$ in HII regions have been found elsewhere.

The main features of the Cygnus-X HII region are summarized in Table II and they are compared with the properties of other HII regions. $T_{S}$ is the 1400 Mcps brightness temperature (undiluted by the aerial

\footnotetext{
${ }^{11}$ W. A. Hiltner and H. L. Johnson, Astrophys. J. 124, 367 (1956)

${ }_{12}$ B. Strömgren, Astrophys. J. 89, 526 (1939).
} 
pattern) of the HII region. Its density would appear to be lower than, and its dimensions greater than, that of more familiar HII regions.

\section{B. PHYSICAL CONDITIONS WITHIN INTER- STELLAR DUST AND GAS CLOUDS}

\section{(1) Observations}

The 21-cm drift curves ${ }^{13}$ were taken across regions in Auriga and Cygnus using a band width of $18 \mathrm{kcps}$ $(3.8 \mathrm{~km} / \mathrm{sec})$ and a beam width of $1.6^{\circ}$. Transits around the region in Auriga are shown in Fig. 2; the recording frequency is displaced $+7 \mathrm{~km} / \mathrm{sec}$ relative to the spiral arm in that direction. The fall in temperature coincides in position $\left(\delta=40.5^{\circ}\right.$ and R.A. $\left.=5^{h} 06^{m}\right)$ and extent $\left(3^{\circ} \times 0.7^{\circ}\right)$ with a dust cloud showing 0.25 magnitude of absorption. Further information about the region comes from spectra taken at the position of the temperature minimum and nearby (the spectrum expected at the position of the source). The difference between these two spectra, $T_{D}(\nu)$, has a half-width of $6 \mathrm{~km} / \mathrm{sec}$ compared with $20 \mathrm{~km} / \mathrm{sec}$ for the emission from the spiral arm.

Similar drift curves were taken in the region R.A. $=19^{h}-23^{h}$ and $\delta=25^{\circ}-55^{\circ}$. They were taken at sample frequencies in the first spiral arm and revealed 6 elongated regions of low brightness temperature whose positions are shown in Fig. 3. The central frequency of each region was different, indicating different distances (or radial velocities) for the clouds. Surveys of obscuring matter in this part of the sky have been made by Pannekoek, ${ }^{14}$ Miller, ${ }^{15}$ and Franklin. ${ }^{16}$ They find elongated obscuring clouds whose positions coincide with the low temperature regions found in the radio survey. The obscuration is greater than $1^{m}$ and in some cases is $4^{m}$. Moreover the optical clouds are remote and this is confirmed by their $21-\mathrm{cm}$ Doppler shift.

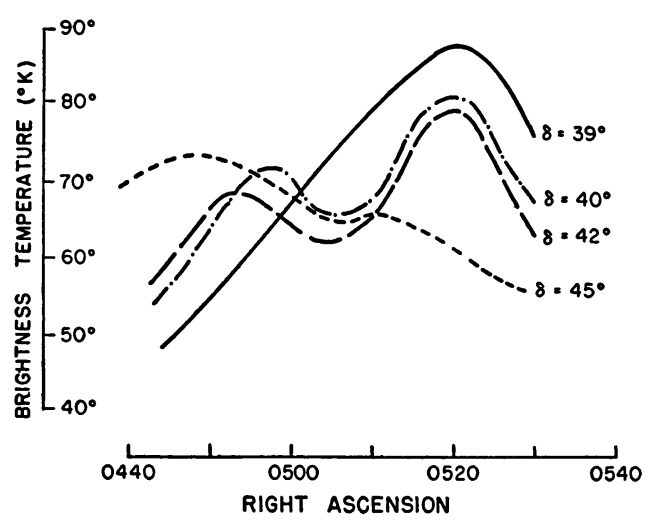

Fig. 2. 21-cm transits near and through the cool cloud in Auriga $\left(\delta=40.5^{\circ}\right.$ and R.A. $\left.=05^{h} 06^{m}\right)$.

${ }^{13}$ R. D. Davies, Monthly Notices Roy. Astron. Soc. 116, 443 (1956).

${ }_{14}$ A. Pannekoek, Publs. Astron. Inst. Univ. of Amsterdam, No. 7 (1942).

${ }_{15}$ F. D. Miller, Harvard Annals 105, 297 (1937).

${ }^{16}$ F. A. Franklin, Astron. J. 60, 351 (1955).

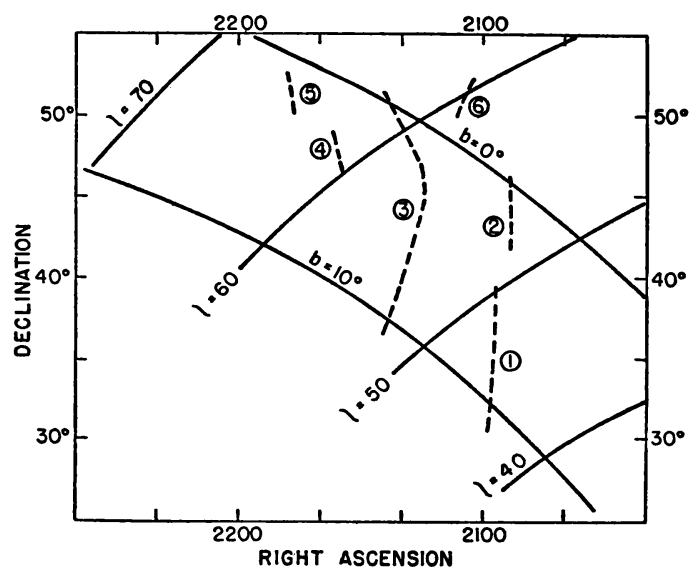

FIG. 3. Map showing the position of cool neutral hydrogen clouds in the vicinity of the constellation of Cygnus.

These $21-\mathrm{cm}$ results can be adequately explained in terms of cool and dense neutral hydrogen regions lying between the bulk of the galactic neutral hydrogen emission and the sun. These regions or clouds absorb the background emission and radiate at a lower brightness temperature than their surroundings.

\section{(2) Temperature of the Clouds}

The kinetic temperature of a cloud can be estimated from its difference spectrum $T_{D}(\nu)$ since

$$
T_{D}(\nu)=\left[T_{B}(\nu)-T_{K}\right]\left[1-e^{-\tau(\nu)}\right],
$$

where $T_{B}(\nu)$ is the brightness temperature of the background emission, $T_{K}$ is the kinetic temperature of the cloud, and $\tau(\nu)$ is its optical depth at a frequency $\nu$. $T_{K}$ is given by the value of $T_{B}(\nu)$ which makes $T_{D}(\nu)$ zero (provided $\tau(\nu)$ remains nonzero). The value of $T_{K}$ for the Auriga cloud is $60^{\circ} \mathrm{K}$.

In the absence of the difference spectrum of a cool cloud, an upper limit for its kinetic temperature is given by the observed brightness temperature at its center. These maximum values of $T_{K}$ for the Cygnus clouds ranged from 25 to $60^{\circ} \mathrm{K}$.

The observed fall in kinetic temperature in dense clouds is that expected from the considerations of Spitzer ${ }^{17}$ and Kahn. ${ }^{18}$ If it is assumed that the proportions of the constituents of interstellar clouds remain the same, then on increasing the density of neutral hydrogen, the dust and gas density will increase linearly although the density of molecular hydrogen (the cooling agent) will increase as the square of the density of the neutral hydrogen. If an average cloud of kinetic temperature $125^{\circ} \mathrm{K}$ has its density increased by 4 or 20 its temperature would fall to $60^{\circ}$ or $40^{\circ} \mathrm{K}$, respectively.

${ }^{17}$ L. Spitzer, Astrophys. J. 107, 6 (1948)

${ }^{18} \mathrm{~F}$. D. Kahn, Gas Dynamics of Cosmic Clouds (Interscience Publishers, Inc., New York, 1955), p. 273. 
TABLE III.

\begin{tabular}{ll}
\hline \multicolumn{1}{c}{ Interstellar cloud } & $\eta(\mathrm{km} / \mathrm{sec})$ \\
\hline Auriga & 5 \\
Cygnus (6 clouds) & 6.5 \\
Cassiopeia (3 clouds) & 3 \\
Sagittarius & 3.5 \\
Optical (6 clouds) & 4.0 \\
Thermal broadening at $100^{\circ} \mathrm{K}$ & 1.9 (HI) \\
& 0.3 (CaII) \\
\hline
\end{tabular}

\section{(3) Velocity Disperions within Clouds}

The velocity dispersions $(\eta)$ within neutral hydrogen clouds will be defined as the half-width of the halfintensity points of the $\tau(\nu)$ spectrum. For the Auriga cloud the maximum optical depth, $\tau_{0}$, is 1 as derived from Eq. (5). $\eta$ is found to be $5 \mathrm{~km} / \mathrm{sec}$. The optical depth of the clouds in Cygnus will also be about unity and the mean value of $\eta$ is $6.5 \mathrm{~km} / \mathrm{sec}$; these values are given in Table III.

There are two other sets of measurements which give us the velocity dispersion within neutral hydrogen clouds. Hagen et al. ${ }^{7}$ have observed the absorption spectrum of the Cassiopeia A radio source with a band width of $8 \mathrm{kcps}$. Assuming that the 3 observed absorption peaks are due to 3 discrete neutral hydrogen clouds, $\eta$ can be derived for each. The mean value is 3 $\mathrm{km} / \mathrm{sec}$. Heeschen ${ }^{8}$ has investigated the "dip" in $21-\mathrm{cm}$ spectra taken in the direction of the galactic center.
On the assumption that this is caused by a nearby cool HI cloud he derives a value of $\eta=3.5 \mathrm{~km} / \mathrm{sec}$. Recent optical measurements (Spitzer and Skumanick ${ }^{19}$ ) of the velocity dispersion within gas clouds indicate similar velocities.

All these results indicate velocities greater than those expected from thermal broadening alone (cf. last entries in Table III). Some of the broadening may be due to expansion effects; however the maximum expansion velocity $\left(\mathrm{Pack}^{20}\right)$ is three times the speed of sound in the gas, and the observed velocity spread for the entire cloud would be much less than this. Hence, the main part of the velocity dispersion within interstellar gas clouds would appear to be the result of turbulence.

\section{(4) Density of Clouds}

From the optical depth and dimensions of the neutral hydrogen clouds in Cygnus and Auriga, an estimate can be made of the neutral hydrogen density using Eq. (2). The density for the Auriga cloud is $250 \mathrm{~cm}^{-3}$, while an estimate for the clouds in Cygnus gives 50 to $100 \mathrm{~cm}^{-3}$. These values may be compared with the average density found within spiral arms, $2 \mathrm{~cm}^{-3}$. High values of neutral hydrogen density within dense dust clouds have also been found by Heeschen.

${ }^{19}$ L. Spitzer and A. Skumanick, Astrophys. J. 116, 452 (1953).

${ }^{20}$ D. C. Pack, Monthly Notices Roy. Astron. Soc. 113, 43 (1953).

\section{DISCUSSION}

G. MÜNCH, Department of Astrophysics, California Institute of Technology, Pasadena, California: To evaluate the dimensions of the Cygnus- $X$ source, you equate the lengths appearing in the expressions for the emission measure and for the absorption produced by the neutral hydrogen. Such a procedure implicitly assumes that the emission region has not modified the kinematics of the surrounding cool region. Now, the recent work on Orion by Menon at Harvard, and my work on the HII regions in the Northern Milky Way, show that the motion of the cool material is affected by the pressure gradient existing between the two regions.

R. D. DAvIES, Jodrell Bank Experimental Station, Manchester University, Manchester, England: Insofar as
I understand the results you refer to, they indicate, (1) a deficiency of neutral hydrogen within the boundary of the HII region, and (2) a concentration of neutral hydrogen at the boundary of the HII region. The first result is precisely that found within the boundary of the Cygnus-X HII region. The second result has no observable counterpart in the Cygnus- $X$ region as far as I could detect. This is perhaps not surprising, since the Cygnus$\mathrm{X}$ HII region is of much greater extent and of lower density than normal HII regions. Moreover, the ionizing stars would appear to be scattered throughout the Cygnus- $\mathrm{X}$ region, and not concentrated in the center as in your case. The kinematics of the two types of region are thus likely to be different. 